\title{
A statistical study of unstable particle populations in the global ring current and their relation to the generation of high $m$ ULF waves.
}

\author{
L. J. Baddeley ${ }^{1}$, T. K. Yeoman ${ }^{1}$, D. M. Wright ${ }^{1}$, K. J. Trattner ${ }^{2}$, and B. J. Kellet ${ }^{3}$ \\ ${ }^{1}$ Department of Physics and Astronomy, University of Leicester, University Road, Leicester LE1 7RH, UK \\ ${ }^{2}$ Lockheed Martin ATC, Palo Alto, USA \\ ${ }^{3}$ Rutherford Appleton Laboratory, Didcot, UK
}

Received: 20 April 2004 - Revised: 18 October 2004 - Accepted: 21 October 2004 - Published: 22 December 2004

\begin{abstract}
The first statistical study of the unstable proton populations which contain "free energy" required to drive small-scale poloidal mode ULF waves in the magnetosphere between L-shell locations of 6 and 9 is presented. The data examined are all in the form of Ion Distribution Functions (IDFs) covering a particle energy range of $0.025 \mathrm{keV}$ to $328 \mathrm{keV}$, amassed over 2.5 years from the TIMAS and CAMMICE (MICS) instruments on-board the Polar spacecraft. Any free energy which is available to drive a resonant wave mode manifests itself as a positive gradient region in the IDF. A new analysis technique applied to the data, allows for the first time, the amount of free energy contained in each IDF to be quantified. The results show that IDFs are a common occurrence in the magnetosphere at these L-shells, although they are most common in the dawn/pre-noon sector. Lower energy (10-45 keV) protons are the most commonly observed unstable populations and also contain the largest amounts of free energy $\left(>10^{10} \mathrm{~J}\right)$. Positive gradient regions at higher energies $(>100 \mathrm{keV})$ are rarely observed and also contain greatly reduced free energies $\left(<10^{9} \mathrm{~J}\right)$.
\end{abstract}

Key words. Magnetospheric physics (Energetic particles, trapped; MHD waves and instabilities) - Space plasma physics (wave-particle interactions)

\section{Introduction}

Ultra Low Frequency (ULF) waves can be classified into two basic types with respect to their generation mechanisms. The first type are characterised by the small azimuthal wave numbers $m$, (or equivalently their large-scale size in the azimuthal direction) and have an energy source external to the Earth's magnetosphere. They are generated by mechanisms such as the Kelvin-Helmholtz instability on the magnetopause, solar wind buffeting or an impulsive increase in solar wind pressure at the front of the magnetopause. The second type of waves are conversely characterised by their high magnitude

Correspondence to: L. J. Baddeley

(lisa.baddeley@ion.le.ac.uk) $m$ numbers. One of the generation mechanisms thought to be responsible for these waves is internal to the magnetosphere in the form of wave-particle interactions between standing wave modes along the geomagnetic field and unstable particle populations in the magnetospheric ring current.

The ring current can be thought of as a toroidal shaped electrical current that flows westward around the Earth. It is located between $\sim 2$ and $9 \mathrm{R}_{E}$, depending on the geomagnetic activity and is made up primarily of hot plasma (tens of $\mathrm{keV}$ to hundreds of $\mathrm{keV}$ ) of solar wind origin (mainly protons, although there are some ionospheric constituents) that has been injected inwards from the tail. The average ring current density can very between $\sim 1-4 \mathrm{nA} \mathrm{m}^{-2}$ (e.g. De Michelis et al., 1997) during quiet intervals to $\sim 7 \mathrm{nA} \mathrm{m}^{-2}$ (e.g. Lui et al., 1987) during stormtime conditions. This corresponds to energy densities of between $\sim 10 \mathrm{keV} \mathrm{cm}^{-3}$ and $\sim 100 \mathrm{keV} \mathrm{cm}^{-3}$. Due to the low plasma densities (and hence small collision frequencies) any injected plasma populations thermalize only on very long time scales. This can give rise to the possibility of the plasma distribution becoming inverted at some point. These "bump-on-tail" or nonMaxwellian ion distribution functions (IDFs) contain "free energy" which manifests itself as a positive gradient region, i.e. $d f / d W$, where $f$ represents the particle distribution function and $W$ the particle energy. It has been suggested (e.g. Southwood, 1976; Hughes et al., 1978) that free energy is fed from the IDFs into a resonant wave mode through inverse Landau damping if, subsequent to an injection into the ring current, the ions gradient-curvature drift westwards and match the local resonance condition (Southwood et al., 1969),

$\omega_{\text {wave }}-m_{\text {wave }} \omega_{\text {drift }}=N \omega_{\text {bounce }}$,

where $N$ is an integer (usually \pm 1 ) and $\omega_{\text {wave }}, \omega_{\text {bounce, }}$, and $\omega_{\text {drift }}$ are the angular frequencies of the wave, the proton bounce, and the proton azimuthal drift, respectively.

There are many different theories (e.g. Southwood et al., 1969; Hasegawa et al., 1971) pertaining to how particle energy is fed into the resonant wave mode. 
However, it is well recognised in all the theories that magnetospheric ion populations are the major energy source for high $m$ pulsations. The different theories are associated with the different states of the ambient plasma in the ring current in the vicinity of the wave and also detail varying wave symmetry requirements and interacting particle population energies. It is presently unclear whether any one generation mechanism and thus particle population is dominant in the generation of westward propagating, high $m$ (i.e. $|m|>10)$ poloidal mode waves in the magnetosphere. This fact was highlighted in a report from the 1997 IAGA (International Association of Geomagnetism and Aeronomy) meeting where it was noted that "in particular, it is important to examine the distribution functions of energetic particles associated with the wave events", Takahashi (1998). Motivated by this, this paper presents a detailed investigation into the energetics of the driving particle populations in the magnetosphere.

Knowledge of the particle energies involved in any resonant interaction will place constraints on the specific wave eigenmode into which energy may be fed. Additional knowledge of the wave characteristics then offers an unambiguous determination of the wave-particle interaction driving the wave. This is usually only possible when conjugate wave and particle data are used in combination. For a given wave frequency $\omega_{\text {wave }}$ and azimuthal $m$ number there are a number of solutions, as a consequence of the resonance equation (Eq. 1), each relating to a different wave mode. These solutions reveal that in general a resonant interaction with a fundamental standing wave structure requires particles of higher energies than with a second harmonic structure.

The ULF waves thought to be generated through the process of inverse Landau damping have been characterised by several different instruments in between $L$-shell locations of 6-9. The location maps to the orbit of geostationary spacecraft which have been utilised in the study of high $m$ waves (e.g. Anderson et al., 1993; Takahashi et al., 1987). It also covers the $L$-shells of ground-based experiments using several high-latitude radar systems such as STARE (the Scandinavian Twin Auroral Radar Experiment; Greenwald et al., 1978; e.g. Allan et al., 1982, 1983), SABRE (the Sweden And Britain auroral Radar Experiment; Nielsen et al., 1983; e.g. Yeoman et al., 1992), BARS (Bistatic Auroral Radar System; McNamara et al., 1983; e.g. Grant et al., 1992) and CUTLASS (Co-Operative UK Twin Located Auroral Sounding System, Milan et al., 1997; e.g. Baddeley et al., 2002) and also the new DOPE HF system (DOppler Pulsation Experiment, Wright et al., 1997; e.g. Yeoman et al., 2000), which have made observations of these waves at a wide variety of MLT locations.

This paper will detail a statistical study of magnetospheric particle data to discover the prevalence of these "bump-ontail" particle distributions which can feed free energy into a resonant wave mode. Such a study has never before been undertaken and so this study will establish the general importance and occurrence rates of such wave-particle interactions in the $L=6-9$ region.

\section{Instrumentation}

The database used for the study comprises omnidirectional IDFs measured using the TIMAS and CAMMICE (MICS) particle detectors on board the Polar spacecraft. The spacecraft was launched on 24 February 1996 with the objective of making both high- and low-altitude measurements of the Earth's polar regions. The orbit is highly elliptical, with apogee at 9 Earth radii $\left(\mathrm{R}_{E}\right)$, perigee at $1.8 \mathrm{R}_{E}$ geocentric and an inclination of $86^{\circ}$. The orbital period is about $18 \mathrm{~h}$, with a spacecraft spin period of $6 \mathrm{~s}$. Initially, apogee was over the northern polar region, but apogee has been moving towards the equator at about $18^{\circ}$ per year. Figure 1 shows 6 single orbits of the spacecraft, each separated by one month, from February 1997 to July 1997 in solar magnetic (SM) co-ordinates. Figure 1a indicates the precession of the orbit as viewed in the $\mathrm{X}-\mathrm{Y}$ plane and shows how the spacecraft can cover all MLTs. Figures $1 \mathrm{~b}$ and $\mathrm{c}$ indicate how the orbit precesses in 6 months in the $\mathrm{X}-\mathrm{Z}$ and $\mathrm{Y}-\mathrm{Z}$ plane. The spacecraft's orbit is fixed with respect to the Sun, and thus traverses north to south in the daytime magnetosphere during autumn and vice versa during spring. The spacecraft spin axis is normal to the orbital plane, to allow a despun platform for the auroral imagers and to enable the particle instruments to map out the full $4 \pi$ steradian distribution functions. The TIMAS instrument (Shelley et al., 1995) measures the lower energy protons from $\sim 0.025 \mathrm{keV}$ to $32 \mathrm{keV}$, while the CAMMICE (MICS) (Wilken et al., 1992) instrument extends to higher energies, $1 \mathrm{keV}$ to $328 \mathrm{keV}$. Both instruments utilise logarithmically spaced energy bins, resulting in wider energy bins at higher particle energies. The original energy limit of CAMMICE (MICS) was $448 \mathrm{keV}$, however, problems with the instrument reduced this range to $328 \mathrm{keV}$.

\section{Free energy calculation}

To determine if a particle population induces wave growth or damping its particle distribution function $f$, must be analysed as a function of particle energy, $W$,

$\frac{d \mathrm{f}}{d \mathrm{~W}}=\frac{\partial \mathrm{f}}{\partial \mathrm{W}}+\frac{d \mathrm{~L}}{d \mathrm{~W}} \frac{\partial \mathrm{f}}{\partial \mathrm{L}}$,

where $L$ is the $L$-shell location of the particles (e.g. Southwood, 1980). Equation (2) takes into account both spatial and energy gradients in the particle population. The gradient of the particle distribution function governs the direction of energy transfer. A negative gradient, $d f / d W<\sim 0$, implies that energy will be transferred from the wave to the particles, inducing wave Landau damping, while $d f / d W>0$ implies that energy may be transferred from the particles to the wave, thus inducing wave growth. This technique allows for the identification of any populations that contain free energy which manifest themselves as a "bump" or positive gradient region, $d f / d W>0$, in the IDF, which elsewhere follows a monotonic power law distribution. 
If this occurs in a region where the particle azimuthal drift velocity is such that it may become phase-locked with the wave azimuthal phase velocity, then free energy is available to the wave and thus wave growth may occur.

The spatial gradient quantity $\mathrm{d} L / \mathrm{d} W \propto m / \omega$ (Chisham, 1996) and thus it can be seen that if $m$ is large and $\omega$ is small (such is the case for the small-scale poloidal mode waves in the magnetosphere), conditions will be more favourable for a larger positive gradient distribution. As shown in Eq. (2) the gradient of the distribution function depends upon both the spatial and energy distributions, both of which will contribute to spacecraft observations of the IDF. Here we detail a new method to estimate the free energy available utilising the quantities measurable by spacecraft traversing the ring current.

The IDFs measured by both TIMAS and CAMMICE (MICS) used here are omnidirectional in nature. A particle population in a given finite velocity range (and therefore a given energy range) will occupy a volume in velocity space $\left(\mathrm{km}^{-6} \mathrm{~s}^{3}\right)$ given by

$\operatorname{Vol}_{V}=\frac{4 \pi}{3}\left(v_{u}^{3}-v_{l}^{3}\right)$

where $v_{u}$ and $v_{l}$ indicate the upper and lower particle velocities of the population. The particle energies are such that the system can be considered as being non-relativistic; thus, the energy is related to the velocity of the particles using $W=\mathrm{mv}^{2} / 2$. The volume in velocity space can then be considered in terms of particle energy,

$V o l_{V}=\frac{4 \pi}{3}\left(\frac{2}{m}\right)^{\frac{3}{2}}\left(W_{u}^{\frac{3}{2}}-W_{l}^{\frac{3}{2}}\right)$.

Given the finite width of the energy range in each data bin, an average value for the count rate in a particular energy bin can only be obtained, not a point measurement of the count rate of particles of a specific energy. Thus, an average value for the distribution function measured by the detectors and thus the number density of particles which occupy that finite volume of phase space is what is utilized in the calculations here. The distribution function averaged over the finite energy width of the detector bands, $\partial f(\mathbf{r}, \mathbf{v})$, is, by definition,

$\partial f(\mathbf{r}, \mathbf{v})=\frac{\partial n}{V o l_{V}}$,

where $\partial \mathrm{n}$ is the number density in normal space $\left(\mathrm{km}^{-3}\right)$ averaged over the detector bandwidth. Rearranging the above expression and substituting in for $\mathrm{Vol}_{V}$ an expression that relates the particle distribution function in velocity space $\partial \mathrm{f}(\mathbf{r}, \mathbf{v})$ to a number density can be obtained,

$\partial n=\partial f(\mathbf{r}, \mathbf{v}) \frac{4 \pi}{3}\left(\frac{2}{m}\right)^{\frac{3}{2}}\left(W_{U}^{\frac{3}{2}}-W_{L}^{\frac{3}{2}}\right)$.

Each detector energy bin has an upper and lower limit, $W_{u}$ and $W_{l}$, with an average value for the particle energy in that bin of $W_{i}=\left(W_{u}+W_{l}\right) / 2$. Each data point $W_{i}$ has a corresponding average distribution function in velocity space $\partial \mathrm{f}_{i}$. A

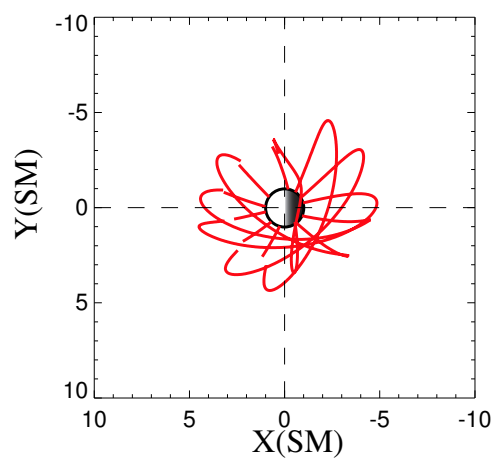

(a)

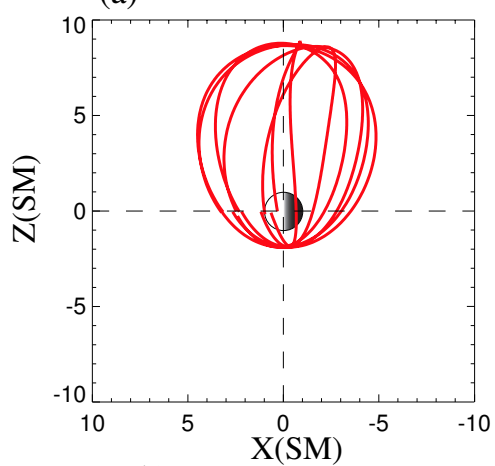

(b)

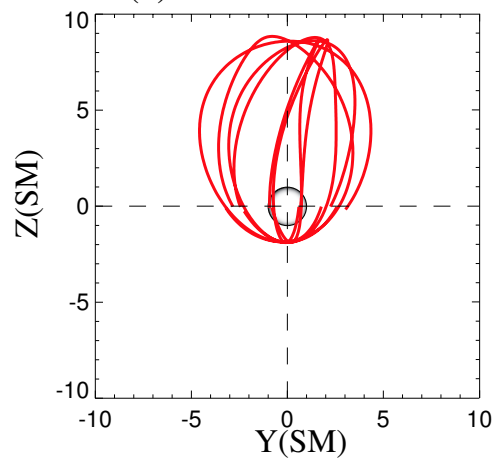

(c)

Fig. 1. Six single orbits of the Polar spacecraft, each separated by one month from February 1997 to July 1997 in solar magnetic (SM) co-ordinates. Figure (a) indicates the precession of the orbit as viewed in the $\mathrm{X}-\mathrm{Y}$ plane (i.e. looking down on the polar cap) and shows how the spacecraft can cover all MLTs. Figures (b) and (c) indicate how the orbit precesses in 6 months in the $\mathrm{X}-\mathrm{Z}$ and $\mathrm{Y}-\mathrm{Z}$ plane, respectively.

sketch of an IDF with a positive gradient region is presented in Fig. 2.

Each $\partial \mathrm{f}_{i}$ data point in the positive gradient region (marked in green on Fig. 2) was then transformed back from velocity space to real space, using Eq. (5), to determine the average number density of particles, $\partial n_{i}$, at that particular energy. It was then assumed that all particles with free energy (i.e. in the positive gradient region) would return to some predefined minimum, $\left(W_{\min }, f_{\min }\right)$ which is set by the location of the initial point of the positive gradient region in each particle distribution (marked point A on Fig. 2). 


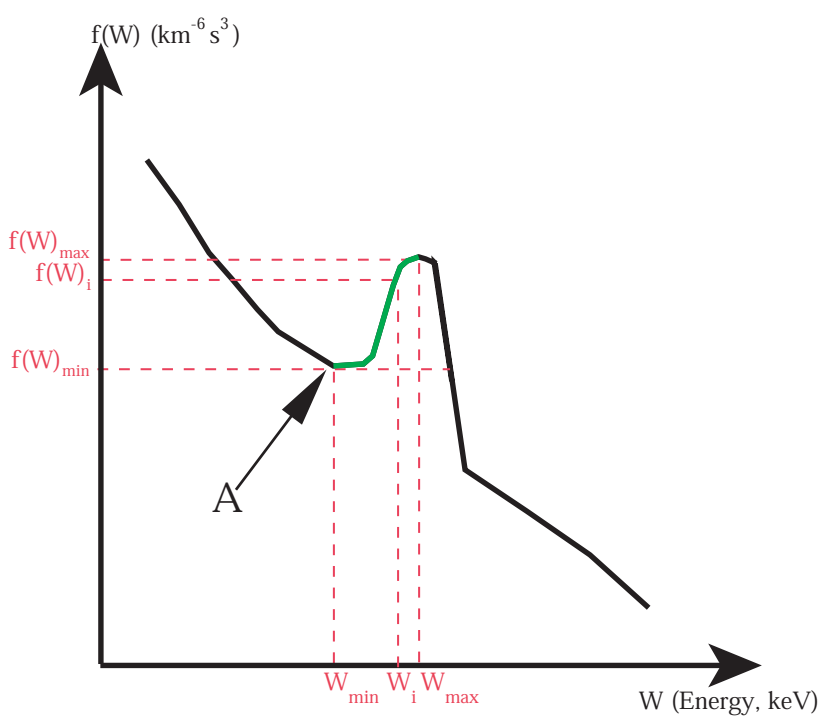

Fig. 2. Schematic indicating how the amount of free energy contained in the positive gradient region is calculated. All data points in the positive gradient region (marked in green) are transposed to a location in phase space marked by point $\mathrm{A}$.

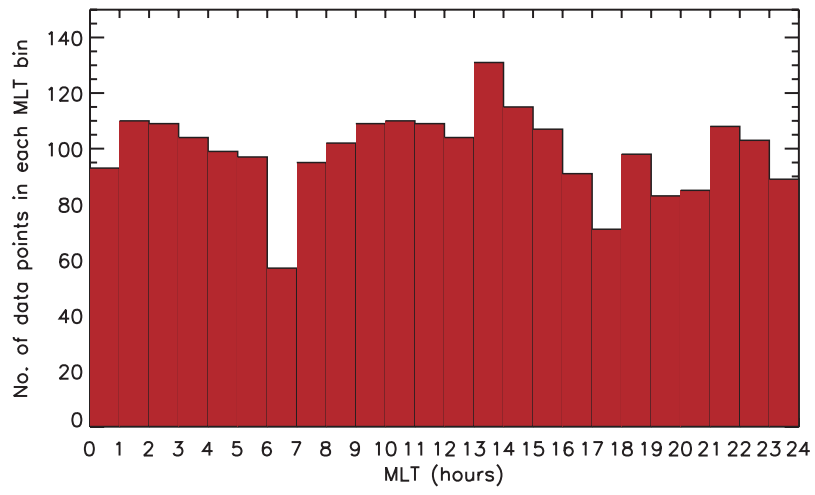

Fig. 3. Histogram of the number of Ion Distribution Functions (IDFs) in each MLT bin.

This can be thought of as a conservative estimate as to the energy release needed for the population to return to a completely monotonic distribution which is given by

$E=\sum_{i}\left(\partial n_{i}-\partial n_{\min }\right)\left(W_{i}-W_{\min }\right)$

This returns an estimate of the energy release in units of $\mathrm{J} \mathrm{km}^{-3}$. By numerical integration of a dipole field an estimate can be made of the corresponding flux tube volume, $V$ which the particle population occupies and thus the total amount of free energy available to a possible resonant wave on that flux tube can be quantified,

$E_{\text {total }}=V * \sum_{i}\left(\partial n_{i}-\partial n_{\min }\right)\left(W_{i}-W_{\min }\right)$

\section{Statistical database}

The database comprises $\sim 2500$ IDFs amassed over a 2.5 year period from May 1996 to December 1998 when the Polar spacecraft was between $L$-shell locations of $\sim 6$ and 9 . The timespan of the data set was chosen due to the limitations in the TIMAS database introduced by telemetry problems and a high voltage breakdown which occurred in the instrument. The data were amassed using the DCR channel of the particle detectors and thus it is assumed that the IDFs were dominated by protons with smaller constituents coming from other heavy ions. The data were first binned into hourly MLT bins to examine the overall data coverage. Figure 3 indicates this coverage for all MLTs in the form of a histogram plot. It can be seen that there is excellent data coverage throughout the magnetosphere, with the distribution maximising at $\sim 13: 00$ MLT. In all there are $\sim 100$ IDFs in each bin from 07:00-16:00 and 18:00-06:00 MLT, with $\sim 70$ data points in each bin in the early morning and late evening sectors.

The populations were investigated, first to ascertain the prevalence of the bump-on-tail IDFs, and second, to quantify the amount of free energy available in the positive gradient region and third to ascertain over what particle energy range it occurs. Various criteria were applied to quantify the characteristics of the particle populations in the magnetosphere. The overlap in energy range between the two instruments allows confirmation and comparison of the measured IDFs to be undertaken, thus, eliminating any possible "false" positive gradient regions due to data spikes or other instrument contamination. The TIMAS instrument has a higher energy resolution at the lower energies and thus data from this instrument was utilized up to particle energies of $21 \mathrm{keV}$. The remaining energy range from $21 \mathrm{keV}$ to $328 \mathrm{keV}$ was covered by the CAMMICE (MICS) instrument. In order to avoid contamination from data spikes a limit was placed on the minimum number of data points which the positive gradient region should contain. In the case of TIMAS this was set to be 4 data points out of a possible 28, each representing one of the 28 energy channels used by TIMAS. Given the lower energy resolution of CAMMICE (MICS) (the higher energy bins span $\sim 20-40 \mathrm{keV}$ ) for this instrument the positive gradient region was required to contain 2 or more data points out of a possible 32. In addition, an upper limit was placed on the magnitude of the slope of the positive gradient, $d f / d W>0$, of $1000 \mathrm{~km}^{-6} \mathrm{~s}^{3}$ to eliminate contamination from data spikes. The final criterion was concerned with the energy of the particles themselves. After consideration of the types of waves that could be driven through wave-particle interactions, it was decided that any positive gradient regions that occurred at an energy of less than $1 \mathrm{keV}$ would not be included in the study. Such factors as the magnitude of the azimuthal $m$ number and frequency of the wave were considered when making the above criterion. Particle driven waves, by their very nature, have high $m$ numbers and frequencies generally in the Pc4 $(\mathrm{f}=7-22 \mathrm{mHz})$ region. 
By utilising the resonance equation (Eq. 1), the characteristics of the types of wave that can be driven by a positive gradient region located below $1 \mathrm{keV}$ may be determined. The waves would either have to have a small $m$ number, which would, in turn, imply that it was not particle driven due to its large spatial size, or, given a large $m$ value, an unrealistic oscillation frequency when considered alongside observational data. Here the average energy location of the positive gradient $\left(W_{a v}=\left(W_{\min }+W_{\max }\right) / 2\right)$ for each IDF is used to determine if the energy location exceeds the $1 \mathrm{keV}$ threshold.

Examples of IDFs from the database are shown in Fig. 4. The plots are in a log-log format with the $\mathrm{x}$-axis indicating the energy of the particles, $W$ from $0.1 \mathrm{keV}$ to $328 \mathrm{keV}$, and the y-axis, indicating the corresponding distribution function, $f(W)$. The blue lines on each plot represent data from the TIMAS instrument, with the red lines representing data from the CAMMICE (MICS) instrument.

It can be seen that there is a reassuringly good agreement between the two instruments. Figure 4a illustrates an IDF without a positive gradient region and thus would not be considered as a candidate for a wave energy source. Figure $4 \mathrm{~b}$ indicates an IDF that would be a suitable wave energy source and thus would be included in the study. The $W_{\min }$ and $W_{\max }$ parameters are indicated in Fig. $4 \mathrm{~b}$. Many of the IDFs contain multiple positive gradient regions. In this situation the positive gradient regions were all included in the study if they were deemed suitable via the selection process described above.

\section{Free energy statistics}

Using the method detailed in Sect. 3 the amount of free energy available was calculated for each IDF. The results are shown in Fig. 5. The results have been divided into hourly MLT bins, depending on the location of the particle population measurement. The graph is orientated such that 06:00 MLT is on the right-hand side, 12:00 MLT at the top, 18:00 MLT on the left-hand side and 24:00 MLT at the bottom. In Fig. 5a the radial distance from the centre of the circle represents the amount of free energy available from 0 to $1.8 \times 10^{11} \mathrm{~J}$ on a linear scale, in increments of $1.2 \times 10^{10} \mathrm{~J}$. In Fig. $5 \mathrm{~b}$ the scale is from 0 to $0.23 \times 10^{11} \mathrm{~J}$ on a linear scale, in increments of $1.2 \times 10^{9} \mathrm{~J}$ allowing, a more detailed look at the structure of the amount of free energy available. No low energy threshold has been employed on the amount of energy contained in the positive gradient region. The amount of free energy was typically $\sim 10^{9} \mathrm{~J}$, with a maximum value of $3.4 \times 10^{11} \mathrm{~J}$ being recorded. The number of occurrences in each energy bin is colour-coded as a percentage of total number of significant IDFs observed in the MLT bin. Each MLT bin has 100 IDFs in it, which implies that there should be no bias in the results arising from data availability. The results in Fig. 5a show that bump-on-tail IDFs are a common occurrence in the magnetosphere. Fifty percent of all IDFs fall into this category at some particle energy range at 06:00 MLT, with this percentage increasing up to $\sim 90 \%$
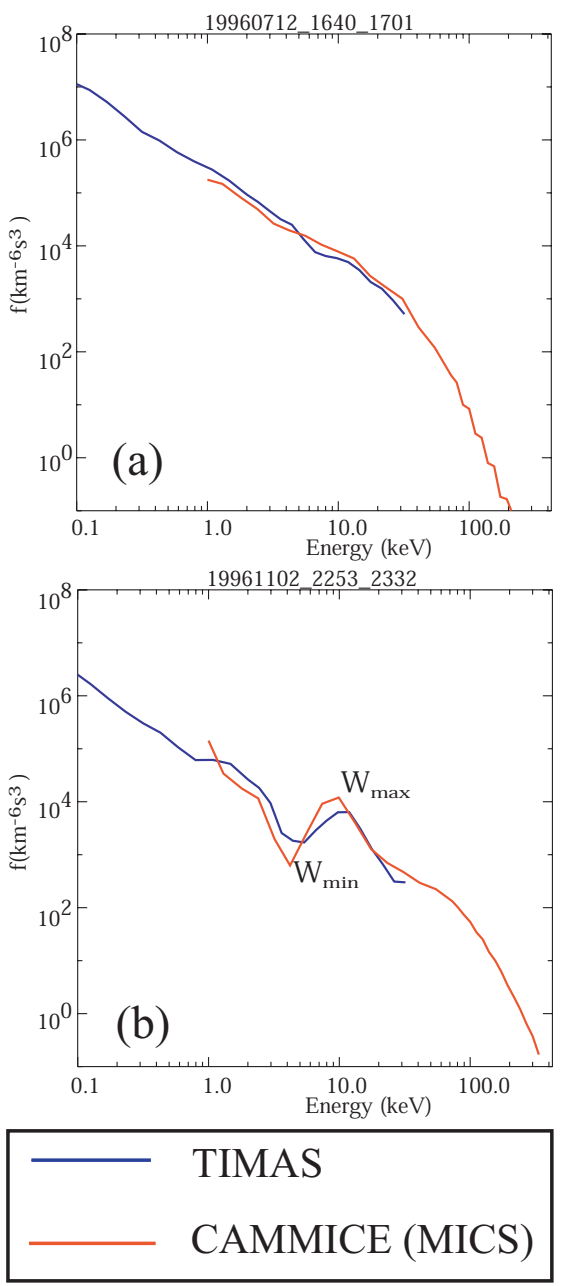

Fig. 4. Example IDFs from the statistical database. TIMAS data is shown in blue and CAMMICE (MICS) data is shown in red. Figure (a) illustrates an IDF without a positive gradient region and thus would not be considered as a candidate for a wave energy source. Figure (b) indicates an IDF that would be a suitable wave energy source and thus would be included in the study. Two further parameters are indicated in Fig. (b) which are later used to indicate over what particle energy range the positive gradient region and thus free energy is located; $W_{\min }$ and $W_{\max }$. $W_{\min }$ indicates the lower energy location of the positive gradient region, while $W_{\max }$ indicates its upper energy limit. For a further explanation of the use of $W_{\min }$ and $W_{\max }$ in calculations, refer to the main text.

for particle populations around 12:00 MLT before decreasing again to $\sim 30 \%$ at 18:00 MLT. Continuing to the nightside (18:00-06:00 MLT) magnetosphere, 30\% of IDFs contain a positive gradient region between $\sim 18: 00$ and 23:00 MLT. From 23:00 to 06:00 MLT this figure increases again to between 35 and $60 \%$.

The overall distribution of the particle population indicates that bump-on-tail distributions tend to be more common in the dayside (06:00-18:00 MLT) magnetosphere and furthermore, more prevalent in the pre-noon than post-noon sector. 

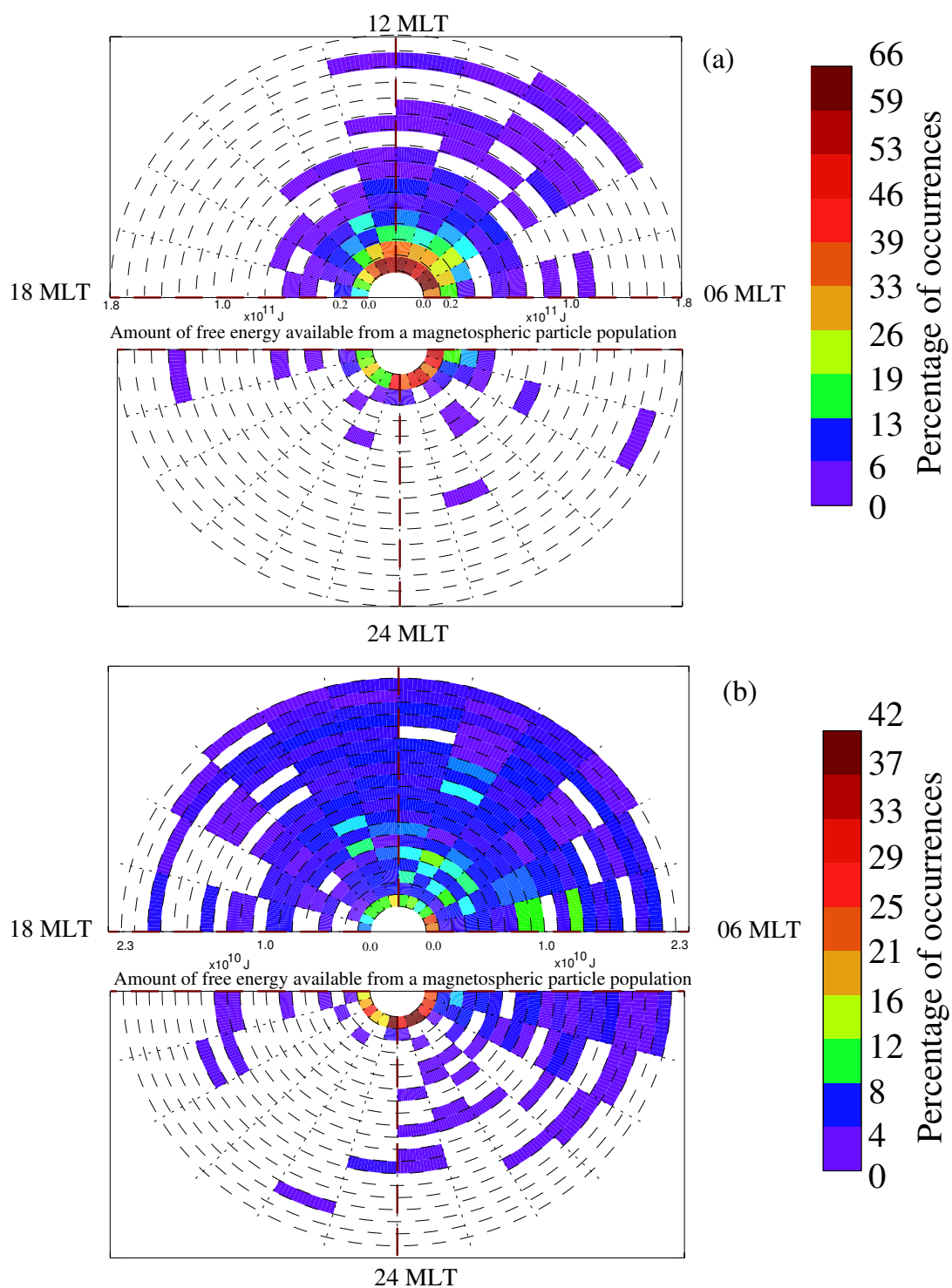

(b)

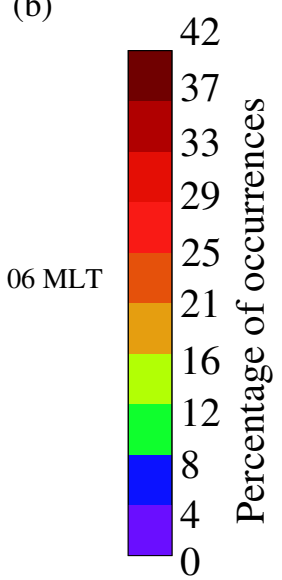

Fig. 5. Results from a statistical study of magnetospheric particle populations, using the TIMAS and CAMMICE (MICS) instruments. Two years of data were binned according to MLT location and also according to the amount of free energy each IDF contained in the form of a positive gradient region. The radial distance from the centre of the circle indicates increasing amounts of free energy available up to a maximum of (a) $1.8 \times 10^{11} \mathrm{~J}$ in bin increments of $1.2 \times 10^{10} \mathrm{~J}$ and $(\mathbf{b})$ up to a maximum of $0.23 \times 10^{11} \mathrm{~J}$ in bin increments of $1.2 \times 10^{9} \mathrm{~J}$. The data bins are colour coded as a percentage occurrence in that MLT bin.

When considering larger amounts of free energy $\left(>1 \times 10^{11} \mathrm{~J}\right)$ the pre-noon sector population is dominant with IDFs containing this larger amount concentrated between 08:00 and 13:00 MLT. What is also of interest is that the occurrence of this increased amount of free energy is rare. Only 10:00-15:00\% of particle populations in each MLT bin measured between 08:00 and 13:00 MLT contain a positive gradient region with this increased amount of free energy. In contrast, Fig. 5b shows that the nightside population as a whole contains less free energy than the dayside population, with $\sim 20-40 \%$ of IDFs in every MLT bin, indicating that $<1.2 \times 10^{9} \mathrm{~J}$ are available.
As noted in Sect. 5 some of the IDFs contained multiple positive gradient regions. If the drift bounce resonance condition (Eq. 1) was satisfied and there were suitable levels of free energy in each of the positive gradient regions, then the possibility exists of multiple wave generation. In such situations either multiple waves at a given harmonic but with different $m$ numbers, or multiple waves at different harmonics but with the same $m$ number, could be generated. 


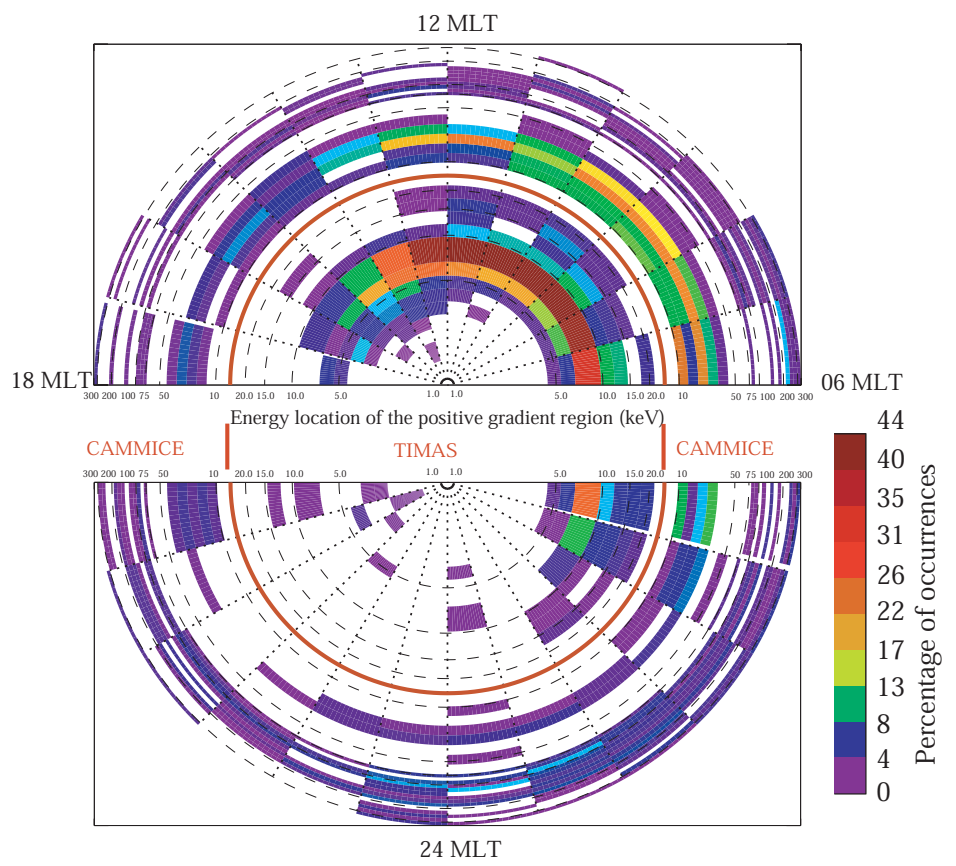

Fig. 6. Results of the study into the energy of the particles which contain free energy. As the radial distance from the centre of the circle is increased the energy of the particles which possess free energy (the $W_{a v}$ parameter) increases. TIMAS data were examined for positive gradient regions between 1 and $21 \mathrm{keV}$ and CAMMICE (MICS) data were examined in a similar manner for regions between 10 and $325 \mathrm{keV}$. The scale is logarithmic and the bin sizes are that of the instruments. The red line indicates the divide between the two instruments. Note there is an energy overlap between the two instruments. The data were binned according to MLT location. The data bins are colour coded as a percentage occurrence in that MLT bin.

\section{Energy of particles in the positive gradient region}

The energy of the particles which may resonantly drive a wave was also examined by investigating the location on the $\mathrm{x}$-axis of the IDF of the positive gradient region (i.e. the $W_{a v}$ parameter). Particle driven ULF waves have been linked to driving particle populations of between tens and hundreds $\mathrm{keV}$, depending on the resonant wave mode. By examining the IDFs in a statistical fashion the prevalence of such particles can be ascertained and thus provide evidence as to the statistical likelihood of any wave modes being driven by these particles in both the pre- and post-noon sectors.

The results (shown in Fig. 6) have again been divided into hourly MLT bins, depending on the location of the particle population measurements and the graph is orientated in the same fashion as Fig. 5. In this part of the analysis, the data from the TIMAS and the CAMMICE (MICS) instrument were dealt with separately. The red semicircular line indicates the divide between the TIMAS and CAMMICE (MICS) data, with TIMAS data located in the inner semicircles. The radial distance from the centre of the circle represents the energy of the particles which possess free energy (the $W_{a v}$ parameter). TIMAS data were examined for positive gradient regions between 1 and $21 \mathrm{keV}$ and CAMMICE (MICS) data were examined in a similar manner for regions between 10 and $325 \mathrm{keV}$. The overlap in energy was utilised to provide additional confirmation that any positive gradient regions were real and not an artefact of the particle detectors. The data bin sizes on the graph are the actual detector bin sizes and the plot is in a log format to allow the full energy range of both detectors to be covered. The bins are colour coded as a function of percentage of the number of IDFs in each MLT bin. It is encouraging to see that in the overlap energy range of $\sim 10-20 \mathrm{keV}$, both TIMAS and CAMMICE (MICS) are in excellent agreement. On the dayside, both instruments indicate that the majority of IDFs have free energy available for a possible resonant interaction with $\sim 10$ $40 \mathrm{keV}$ protons. This trend is strongest in the dawn sector with $\sim 60 \%$ of the IDFs falling into this category. The trend continues slightly into the dusk sector, although again, the percentage of IDFs containing free energy is reduced. On the nightside the overall number of occurrences is far less than the dayside. Most of the IDFs here display positive gradient regions at energies of between 75 and $300 \mathrm{keV}$, except at 04:00-06:00 MLT, where the distribution resembles that of the dayside pre-noon distributions. What is most surprising is the small number of IDFs which contain particles with free energy above $\sim 75 \mathrm{keV}$. In all dayside MLT bins $<10 \%$ of the particle populations indicate the presence of $75 \mathrm{keV}$ protons with free energy to feed a possible wave mode. This figure is only slightly larger on the nightside, with up to $\sim 25 \%$ of IDFs in each MLT bin, indicating the presence of a positive gradient region between 75 and $300 \mathrm{keV}$. 
In Fig. 6 all data has been included, regardless of how small the amount of free energy that is available. The authors are unaware of any published data which details with the amount of energy available from an unstable particle population, such as the ones presented here. At this time it is unclear what magnitude of positive gradient region, in terms of the amount of free energy it contains, is significant for driving ULF waves. However, there have been publications which detail the amount of energy dissipated into the ionosphere by the ULF waves which may be driven by the particle populations. Greenwald and Walker (1980) calculated that an externally driven large-scale Pc5 wave dissipated $\sim 10^{13} \mathrm{~J}$ of energy in the ionosphere. Large-scale waves, which are sustained by an external energy source, such as the KelvinHelmholtz instability or solar wind buffeting, clearly involve larger energies than the internally driven waves which are driven through inverse Landau damping. Allan and Poulter (1984) reported observations of two particle driven waves: a storm time Pc5 pulsation and also a Pg using the STARE radar. They estimated that $\sim 10^{10} \mathrm{~J}$ dissipated into the ionosphere for the storm time Pc5 while in the case of the Pg, they ascertained that $\sim 10^{11} \mathrm{~J}$ was dissipated in the ionosphere. Given the estimates obtained by the above authors the particle populations described here were further investigated by increasing the threshold of free energy by two orders of magnitude from $1 \times 10^{9} \mathrm{~J}$ to $1 \times 10^{11} \mathrm{~J}$. The results are shown in Fig. 7 in the same format as Fig. 6. Figure 7a indicates positive gradient regions which contain $>1 \times 10^{9} \mathrm{~J}$, Fig. $7 \mathrm{~b}$ indicates positive gradient regions which contain $>1 \times 10^{10} \mathrm{~J}$ and Fig. 7c indicates positive gradient regions which contain $>1 \times 10^{11} \mathrm{~J}$. By comparing the figures it can instantly be seen that the occurrence of particles with energies of $>75 \mathrm{keV}$ reduces significantly when an energy threshold of $>10^{9} \mathrm{~J}$ is applied. Only $\sim 1 \%$ of the total IDF population measured over the 2.5 years of the statistical study indicate protons of $\sim 100 \mathrm{keV}$ with free energy of $>1 \times 10^{9} \mathrm{~J}$. What is also noticeable is that the $10-40 \mathrm{keV}$ particle populations do contain $>1 \times 10^{9} \mathrm{~J}$, implying that they are more likely to be candidates for wave particle interactions. Additionally, it is only the $10-40 \mathrm{keV}$ particles which contain the larger amounts, $>1 \times 10^{11} \mathrm{~J}$, of free energy. The majority of particles which contain this amount of free energy are clustered in the prenoon dayside magnetosphere. There is, however, a single occurrence of an IDF containing this amount if there is free energy in the 18:00-19:00 MLT bin and also in the 01:0002:00 MLT bin.

\section{Discussion}

The high $m$ ULF waves of interest in this paper derive their energy through inverse Landau damping with nonMaxwellian particle populations in the magnetospheric ring current. The statistical study presented here examined 2.5 years of particle data from the TIMAS and CAMMICE (MICS) particle detectors on board the Polar spacecraft to ascertain the prevalence of such driving ring current particle populations. Such a study has never previously been undertaken. Several interesting conclusions can be drawn about the possible dominant interaction mechanism and thus wave eigenmode at different MLT locations.

\subsection{Morning sector observations}

This study has shown that relatively low energy particles, between $\sim 10$ and $40 \mathrm{keV}$, are the dominant energy source in the magnetospheric ring current, between $L$-shells 6 to 9. Additionally, these lower energy particles contain greater free energies than the higher energy $(\sim 100 \mathrm{keV})$ particles. This study has also shown that the majority of IDFs which do contain such free energy are located in the dawn sector of the magnetosphere. This would imply that the dawn sector should be the most fertile for high $m$ ULF wave generation at these particle energies. Recent work by Yeoman et al. (2000), utilising ionospheric measurements of ULF waves made with a HF Doppler sounder (DOPE), indicated that the pre-noon sector was the dominant region for high $m$ wave generation, where 41 out of a total of 52 events did not display a conjugate ground magnetometer signature in the morning sector.

In contrast, previous statistical studies into radially polarised ULF waves using satellite data (e.g. Arthur and McPherron, 1981; Kokuburn, 1989; Engebretson et al., 1992; Anderson, 1993) have implied that the dusk work, which indicated that the dusk sector was the dominant region for ULF wave generation, did so on the strength of satellite observations of the wave field. The combination of the study presented in this paper and the study by Yeoman et al. (2000) have indicated that it is possible that satellite studies may have provided an incomplete picture. This could be due to the fact that satellites provide data of a limited temporal and spatial extent in the wave excitation region.

The only ionospheric observations of morning sector high $m$ ULF wave and conjugate driving bump-on-tail particle populations were presented by Baddeley et al. (2002) and indicated driving a particle population of energy $\sim 10 \mathrm{keV}$. The authors are unaware of any published conjugate observational evidence of such a bump-on-tail particle population at $\sim 100 \mathrm{keV}$. Publications which have stated that the driving particle population was $\sim 100 \mathrm{keV}$ protons have done so on the basis of observed particle modulations detected in the vicinity of the wave at the wave frequency, or by utilising either spacecraft measurements of the magnetic field in the equatorial plane, or near conjugate, inter-hemispheric ground magnetometer stations, to infer the wave eigenmode.

\subsection{Giant pulsation $(\mathrm{Pg})$ observations}

A major topic of debate in the field of particle driven ULF waves is the energy of the interacting proton populations that drive morning sector waves, particularly Pgs. Previous work has cited 10-40 keV protons (e.g. Wright et al., 2001 and Chisham, 1996) and 100-150 keV protons (e.g. Thompson et al., 2001) as the driving particle populations for Pgs. 

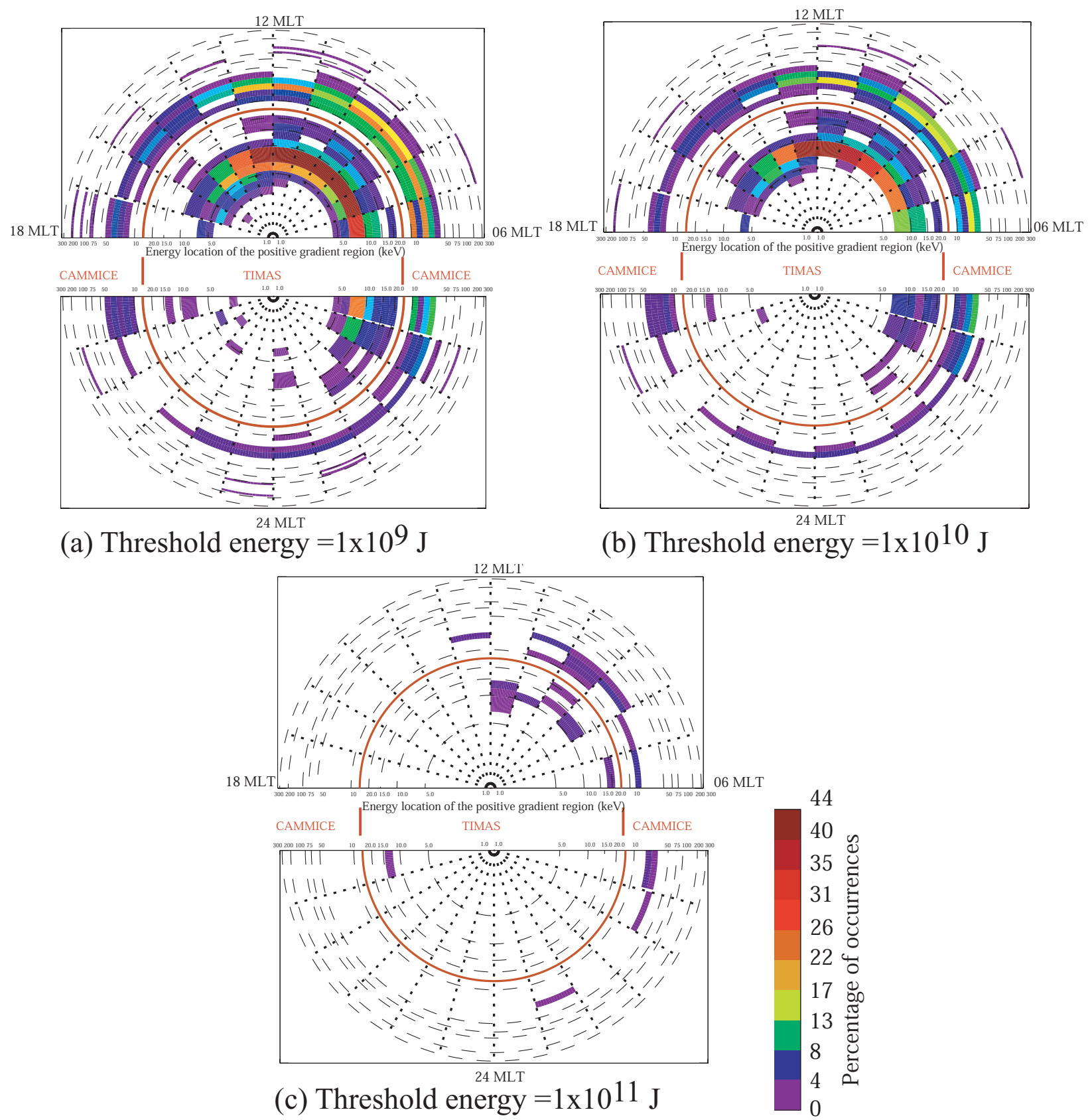

Fig. 7. As for Fig. 6, but with a total free energy threshold applied at (a) $1 \times 10^{9} \mathrm{~J}$, (b) $1 \times 10^{10} \mathrm{~J}$ and (c) $1 \times 10^{11} \mathrm{~J}$. This has the effect of reducing the occurrences at higher particle energies.

Thompson et al. (2001) utilised near conjugate observations of a Pg using magnetometers in Iceland and Antarctica to infer on odd mode standing wave structure. This method of inferring the wave eigenmode, utilizing inter-hemispheric magnetometer data, may be unreliable. Previous work into Pg eigenmodes by Annexstad and Wilson (1968) inferred an even mode structure, while Green (1979) inferred an odd mode structure despite both using this same method. The main problem in this method is the fact that the locations of the magnetometers are judged to be conjugate using in- ternal magnetic field models (e.g. Tsyganenko, 1989). As pointed out by Takahashi et al. (1992) different field models can produce discrepancies as large as $\sim 4^{\circ}$ in longitude between them. When considering Pgs, which have large phase changes over a small longitudinal extent, this difference can result in supposed conjugate magnetometer phase data being wrongly compared, resulting in an inaccurate eigenmode deduction. Thompson et al. (2001) also utilised geosynchronous proton fluxes as measured by the Los Alamos National Laboratory (LANL) spacecraft. 
They noted proton fluctuations in the $104-125 \mathrm{keV}$ and $125-153 \mathrm{keV}$ channels at a frequency identical to the Pg. They concluded that the Pg was the result of a drift resonance interaction between an odd mode standing wave and $\sim 100 \mathrm{keV}$ protons. Similar flux oscillations observed by the AMPTE/ IRM satellite have been noted by Baumjohann et al. (1987) when investigating a post-noon compressional Pc5 event. In their paper they also noted a positive gradient region in the differential energy flux spectra for proton energies of between $\sim 1$ and $30 \mathrm{keV}$. The magnitude of the flux modulations maximised over a similar energy band, between $10-40 \mathrm{keV}$. They concluded that the compressional pulsation had a fundamental mode structure and was driven through a drift resonance interaction, such as the drift mirror mode, (e.g. Pokhotelov et al., 1986) with $40 \mathrm{keV}$ protons. While it is entirely plausible that the wave field was indeed modulating the proton flux, this knowledge alone does not imply that this was the particle population which provided the free energy. Without measurements of the particle distribution function the energy of the driving particle population cannot be conclusively specified. Evidence from this extensive statistical study indicates that not only are $100 \mathrm{keV}$ protons, which contain free energy, very rare in the pre-noon sector, but they also contain very meagre amounts of free energy. Thus, an analysis of the particle population statistics would suggest that it is unlikely that Pgs can be driven through a drift resonance interaction.

There have been only two publications which present conjugate ground-based observations of a Pg and satellite observations of the driving particle population. A recent paper by Wright et al. (2001) reported multi-instrument observations of a Pg pulsation. The wave was detected simultaneously on the ground by the International Monitor for Auroral Geomagnetic Effects (IMAGE) magnetometer network and in the ionosphere by DOPE. Shortly before the commencement of the Pg, the particle instruments on board the Polar spacecraft detected a bump-on-tail proton distribution drifting westward. By solving the resonance equation (Eq. 1) Wright et al. (2001) concluded that the Pg is likely to be the result of a drift-bounce resonance interaction between an even mode standing wave and energetic particles of around $7 \mathrm{keV}$, at the lower energy edge of the observed unstable distribution. Glassmeier et al. (1999) suggested that a resonant interaction with particles of $\sim 67 \mathrm{keV}$ measured by the GOES 2 spacecraft was responsible for driving a Pg observed in magnetometer data from the Scandinavian magnetometer array. In the paper they inferred that the resonance equation could be satisfied by a non-integer value of $N$ if the equation was modified slightly, thus invoking an odd mode fundamental field line oscillation interaction. The comment by Mann and Chisham (2000) which followed suggested that the "real" bump-on-tail was located outside the energy range of the particle detector and that the Pg observed could be driven though either an interaction with $12 \mathrm{keV}$ or $250 \mathrm{keV}$ protons and thus be an even or odd mode standing wave, respectively. The findings of this study support the comments made by Mann and Chisham (2000) and indicate that an in- teraction with $12 \mathrm{keV}$ protons is the more likely statistically, in terms of both the MLT location of the particles and also the amount of free energy available.

The findings of this study also support modelling work undertaken by Ozeke and Mann (2001). Pgs are only observed in the morning sector under geomagnetically quiet conditions (Chisham, 1996). Following on from this work, Ozeke and Mann (2001) calculated the trajectories of protons in a dipole magnetic field and developed a model which would reveal favourable locations in particle energy, $W$ and $L$-shell space for high $m$ wave growth through wave particle interactions. Their model indicated that in the morning sector very monochromatic Pc4 waves, highly localised in latitude, would be generated through a drift-bounce resonance interaction between a second harmonic standing mode wave and $10 \mathrm{keV}$ protons; such waves fit the characteristics of Pgs. The work here also supports that of Chisham (1996), who indicated that Pgs are driven by $5-30 \mathrm{keV}$ protons. He noted that under geomagnetically quiet conditions ions which have been injected into the nightside on closed paths are able to drift to the morning sector. Under more active conditions, the $\boldsymbol{E} \times \boldsymbol{B}$ drifts are large, de-trapping the protons from their closed drift paths, causing them to follow convective paths to the dayside magnetopause, where the particles are lost.

\subsection{Afternoon sector observations}

In the afternoon sector, there appear to be two dominant particle driven wave types: radially polarised Pc4 pulsations which have been observed during geomagnetically quiet times, and compressional Pc5 pulsations, so-called storm time Pc5s, which have been observed during geomagnetically active times. Engebretson et al. (1992) utilised geosynchronous satellite observations to observe 21 pulsation events in the dayside magnetosphere. They concluded that the Pc4 pulsations occurred primarily in the dusk sector and surmised that the waves were driven through a resonant interaction with $100 \mathrm{keV}$ protons. Storm time Pc5 pulsations have been seen in STARE data (Allan et al., 1982, 1983). They are compressional waves of high $m$ number $(m \sim-7$ to -80) which are observed in the dusk sector during magnetically disturbed intervals. The wave onsets are closely associated with enhancements in the ring current as a result of newly injected plasma from the magnetotail (e.g. Engebretson and Cahill, 1981; Allan et al., 1982) and it is agreed that they derive their energy through wave-particle interactions. They have been related to particle energies of between 10 and $70 \mathrm{keV}$, and it is generally agreed that they have an odd mode standing structure and are generated through a drift resonance interaction, (e.g. Allan et al., 1982, 1983).

Similar waves, termed "diamagnetic class" waves have been observed in the dusk sector by Woch et al. (1990), who favoured an even mode standing structure using measurements from the GOES 2 satellite and a drift mirror mode instability, although they did not specify the energy of the interacting particle population. The authors suggest that the waves are related to the classical storm time Pc5s. 
Similar high $m$ waves, but with an equatorward phase motion, have been seen in SABRE and BARS coherent radar systems also in the dusk sector by Yeoman et al. (1992) and Grant et al. (1992), respectively. They also suggested that these waves derive their energy from energetic particle populations.

More recently, Yeoman et al. (2001) have observed high $m$ Pc4 waves in the post-noon sector using the CUTLASS radars. They suggested the waves were driven through both drift and drift-bounce resonance with $37-57 \mathrm{keV}$ and 35 $41 \mathrm{keV}$ protons, respectively. In all cases the authors cited in this section have indicated further research must be undertaken as no one generation mechanism can fully explain their observations. Results from the study presented here indicate that in the post-noon sector there are populations of 10 $50 \mathrm{keV}$ ions which contain free energy. Additionally, from Fig. 7 , the populations do contain significant $\left(>1 \times 10^{10} \mathrm{~J}\right)$ free energy which would identify them as suitable candidates for driving any resonant wave mode. Only $\sim 1 \%$ of the total IDF population measured over the 2.5 years of the statistical study indicate protons of $\sim 100 \mathrm{keV}$ with free energy of $>1 \times 10^{9} \mathrm{~J}$. This figure increases to $\sim 80 \%$ when considering $30-60 \mathrm{keV}$ protons. The results from this statistical study thus support the inferred particle energies of Allan et al. $(1982,1983)$ and also Yeoman et al. (2001).

This study has indicated that very few $\sim 100 \mathrm{keV}$ particles possess significant amounts of free energy to impart to any resonant waves' field line at all MLTs. If these higher energy particles play a role in wave generation, then either they do so under extremely rare occasions or they cannot be detected by current instruments, possibly due to pitch angle effects. The European Space Agency's (ESA) Double Star mission, which involves two spacecraft, one in a polar orbit and one in an equatorial orbit, will allow particle populations at different positions along the field line to be viewed simultaneously, thus allowing for the plasma environment of the wave field to be more thoroughly examined.

As mentioned in Sect. 4, the IDFs analysed in this paper are dominated by protons. The possibility does exist that heavier ions can cause wave growth in the same manner. Chisham and Mann (1999) analysed a Pc5 wave which occurred at $\mathrm{L} \sim 4.5$ and pointed out that energetic $\mathrm{O}+$ ions located at the inner edge of the ring current may, under appropriate conditions, provide the energy needed for wave growth.

\section{Summary}

This paper has presented a statistical study of magnetospheric ring current ion populations using 2.5 years of IDFs. The IDFs were examined to ascertain the statistical likelihood of specific particles containing free energy and also how much free energy the populations contained. A study of this manner has never before been undertaken. This has proved to be an illuminating study for understanding the generation mechanism of high $m$ ULF waves. The results of this study indicate that lower energy $(10-45 \mathrm{keV})$ protons are the dominant non-Maxwellian populations for any interacting wave mode. Not only is the occurrence of these particles dominant, they also routinely contain the largest amount of free energy $\left(>10^{10} \mathrm{~J}\right)$. Free energy associated with particles of higher energies $(>100 \mathrm{keV})$ are rarely observed and also contain greatly reduced amounts of free energy $\left(<10^{9} \mathrm{~J}\right)$. Although this study alone cannot indicate the eigenmode structure of any interacting wave, when combined with previous work certain conclusions can be drawn. More IDFs containing free energy were observed on the dawn sector which, in agreement with the work of Yeoman et al. (2000), indicates that the dawn sector could be a more fertile region for high $m$ ULF wave generation then previously thought. Additionally, this work suggests that Pgs are more likely to be an even mode structure driven by a drift-bounce resonance interaction with $10-45 \mathrm{keV}$ protons. The fact that this study has revealed very few possible sources of free energy from protons with energies $>45 \mathrm{keV}$ suggests that it is unlikely that Pgs can be driven through a drift resonance interaction, such as that cited by Thompson et al. (2001) and Glassmeier et al. (1999). This work supports the work of Allan et al. (1982, 1983), Yeoman et al. (2001) and Baumjohann et al. (1987) in identifying pulsations in the dusk sector such as storm time Pc5 pulsations as fundamental mode oscillations which may be driven by particles in the $30-60 \mathrm{keV}$ energy range in the dusk sector.

As shown in this study, particles of higher energies $(\sim 100 \mathrm{keV})$ are rarely observed to contain significant amounts of free energy $\left(<10^{9} \mathrm{~J}\right)$. It is also possible, however, that the higher energy particles $(\sim 100 \mathrm{keV})$ are not detected by the Polar spacecraft, possibly due to the high-latitude location of the spacecraft which precludes measurements of particles with large pitch angles, which would be confined to the equatorial regions of the magnetosphere. Further investigation is certainly needed utilising multi-satellite particle observations from projects such as Cluster and Double Star.

Acknowledgements. The authors would like to thank T. Fritz, PI of CAMMICE (MICS), and W. Peterson, PI of TIMAS. LJB is supported by a PPARC Quota Studentship.

Topical Editor T. Pulkkinen thanks S. Kokobun and another referee for their help in evaluating this paper.

\section{References}

Allan, W. and Poulter, E. M.: The spatial structure of different ULF pulsation types: A review of STARE radar results, Rev. Geophys. and Space Phys., 22, 85-97, 1984.

Allan, W., Poulter, E. M., and Nielsen, E.: STARE observations of a Pc5 pulsation with large azimuthal wave number, J. Geophys. Res. 87, 6163-6172, 1982.

Allan, W., Poulter, E. M., and Nielsen, E.: Pc5 pulsations associated with ring current proton drifts: STARE radar observations, Planet. Space Sci., 31, 1279-1289, 1983.

Anderson, B. J.: Statistical studies of Pc 3-5 pulsations and their relevance for possible source mechanisms of ULF waves, Ann. Geophys., 11, 128-143, 1993. 
Annexstad, J. O. and Wilson,C. R.: Characteristics of Pg micropulsations at conjugate points, J. Geophys. Res., 73, 1805-1818, 1968.

Arthur, C. W. and, McPherron, R. L.: The Statistical character of Pc4 magnetic pulsations at synchronous orbit, J. Geophys. Res., 86, 1325-1334, 1981.

Baddeley, L. J., Yeoman, T. K., Wright, D. M., Davies, J. A., Trattner, K. J., and Roeder, J. L.: Morning sector drift-bounce resonance driven ULF waves observed in artificially induced HF radar backscatter, Ann. Geophys., 20, 1487-1498, 2002.

Baumjohann, W., Sckopke, N., LaBelle, J., and Klecker, B.: Plasma and Field Observations of a Compressional Pc5 Wave Event, J. Geophys. Res., 92, 12 203-12 212, 1987.

Chisham, G.: Giant pulsations: An explanation for their rarity and occurrence during geomagnetically quiet times, J. Geophys. Res.,101, 24 755-24 763, 1996.

Chisham, G. and Mann, I. R.: A Pc5 ULF wave with large azimuthal wavenumber observed within the morning sector plasmasphere by Sub-Auroral Magnetometer Network, J. Geophys. Res.,104, 14717-14 727, 1999.

Cramm, R., Glassmeier, K. H., Othmer, C., Fornacon, K. H., Auster, H. U., Baumjohann, W., and Georgescu, E.: A case study of a radially polarised $\mathrm{Pc} 4$ event observed by the Equator $-\mathrm{S}$ satellite, Ann. Geophys., 18, 411-415, 2000.

De Michelis, P., Daglis, I. A., and Consolini, G.: Reconstruction of the terrestrial ring current derived from AMPTE/CCE-CHEM, J. Geophys. Res., 102, 14 103-14 112, 1997.

Engebretson, M. J., Murr, D. L., Erickson, K. N., Strangeway, R. J., Klumpar, D. M., Fuselier, S. A., Zanetti, L. J., and Potemra, T. A.: The spatial extent of radial magnetic pulsation events observed in the dayside near synchronous orbit, J. Geophys. Res., 97, 13 741-13 758, 1992.

Engebretson, M. J. and Cahill, L. J.: Pc5 pulsations observed during the June 1972 geomagnetic storm, J. Geophys. Res.,86, 56195631, 1981.

Glassmeier, K. H., Buchert, S., Motschmann, U., Korth, A., and Pedersen, A.: Concerning the generation of geomagnetic giant pulsations by drift-bounce resonance ring current instabilities, Ann. Geophys., 17, 338-350, 1999.

Grant, I. F., McDiarmid, D. R., and McNamara, A. G.: A class of high- $m$ pulsations and its auroral radar signature, J. Geophys. Res.,97, 8439-8451, 1992.

Green, C. A.: Observations of Pg pulsations in the northern auroral zones and at lower latitude conjugate points, Planet. Space Sci., 27, 63-77, 1979.

Greenwald, R. A. and Walker, A. D. M.: Energetics of long period resonant hydromagnetic waves, Geophys. Res. Lett., 7, 745-748, 1980.

Greenwald, R. A., Weiss, W., Nielsen, E., and Thompson, N. R.: STARE: A new radar auroral backscatter experiment in northern Scandinavia, Radio Sci., 13, 1021-1039, 1978.

Hasegawa, A.: Drift-wave instabilities of a compressional mode in high - $\beta$ plasma, Phys. Rev. Lett., 27, 11-14, 1971.

Hillebrand, O., Münch, J., and McPherron, R. L.: Ground-satellite correlative study of a giant pulsation event, J. Geophys. Res.,51, 129-140, 1982.

Hughes, W. J., Southwood, D. J., Mauk, B., McPherron, R. L., and Barfield, J. N.: Alfvén waves generated by an inverted plasma energy distribution, Nature, 275, 43- 44, 1978.

Kokubun, S., Erickson, K. N., Fritz, T. A., and Mcpherron, R. L.: Local time asymmetry of Pc4-5 pulsations and associated particle modulations at synchronous orbit, J. Geophys. Res., 94,
6607-6625, 1989.

Lui, A. T. Y., McEntire, R. W., and Krimigis, S. M.: Evolution of the ring current during two geomagnetic storms, J. Geophys. Res., 92, 7459-7468, 1987.

Mann, I. R. and Chisham, G.: Comment on "Concerning the generation of geomagnetic giant pulsations by drift-bounce resonance ring current instabilities" by K. H. Glassmeier et al. Ann. Geophys., 17, 338, 1999,Ann. Geophysicae, 18, 161-166, 2000.

Milan, S. E., Lester, M., and Moen, J.: A comparison of optical and coherent HF radar backscatter observations of a post-midnight aurora, Ann. Geophys., 15, 1388, 1997.

McNamara, A. G., McDiarmid, D. R., Sofko, G. J., Koeler, J. A., Forsyth, P. A., and Moorcroft, D. R.: BARS - a dual bistatic auroral radar system for the study of electric fields in the Canadian sector of the auroral zone, Adv. Space Res., 2, 145-159, 1983.

Nielsen, E., Guttler, W., Thomas, E. C., Stewart, C. P., Jones, T. B., and Hedburg, A.: SABRE - new radar-auroral backscatter experiment, Nature, 304, 712-714, 1983.

Ozeke, L. G. and Mann, I. R.: Modeling the properties of high $m$ Alfvén waves driven by the drift-bounce resonance mechanism, J. Geophys. Res., 106, 15 583-15 597, 2001.

Pokhotelov, O. A., Pilipenko,V. A., Nezlina, Y. M., Woch, J., Kremser, G., North , A., and Amata, E: Excitation of high $\beta$ plasma instabilities at the geostationary orbit-theory and observations, Planet. Space Sci., 34, 695-712, 1986.

Shelley, E. G., Ghielmetti, A. G., Balsiger, H., Black, R. K., Bowles, J. A., Bowman, R. P., Bratschi, O., Burch, J. L., Carlson, C. W., Coker, A. J., Drake, J. F., Fischer, J., Geiss, J., Johnstone, A., Kloza, D. L., Lennartsson, O. W., Magoncelli, A. L., Paschmann, G., Peterson, W. K., Rosenbauer, H., Sanders, T. C., Steinacher, M., Walton, D. M., Whalen, B. A., and Young, D. T.: The Toroidal Imaging Mass-Angle Spectrograph (TIMAS) for the Polar Mission,Space Sci. Rev., 71, 497-530, 1995.

Southwood, D. J.: A general approach to low-frequency instability in the ring current plasma, J. Geophys. Res.,81, 3340-3348, 1976.

Southwood, D. J., Dungey, J. W., and Etherington, R. J.: Bounce resonant interactions between pulsations and trapped particles, Planet. Space Sci., 17, 349-361, 1969.

Southwood, D. J.: Low frequency pulsation generation by energetic particles, J. Geomag. Geoelec., 32, SII 75, 1980.

Takahashi, K.: ULF waves: 1997 IAGA division 3 reporter review, Ann. Geophysicae, 16, 787-803, 1998.

Takahashi, K., Sato, N., Warnecke, J., Lühr, H., Spence, H. E., and Tonegawa, Y.: On the standing wave mode of giant pulsations, J. Geophys. Res., 97, 10717-10 732, 1992.

Takahashi, K., Fennell, J. F., Amata, E., and Higbie, P. R.: Fieldaligned structure of the storm time Pc5 wave of 14-15 November 1979, J. Geophys. Res., 92, 5857-5864, 1987.

Thompson, S. M. and Kivelson, M. G.: New Evidence for the origin of giant pulsations, J. Geophys. Res.,106, 21 237-21 253, 2001.

Tsyganenko, N. A., A magnetospheric magnetic field model with a warped tail current sheet, Planet. Space Sci., 37, 5-20, 1989.

Wilken, B., Weiß, W., Hall, D., Grande, M., Sørass, F., and Fennell, J. F.: Magnetospheric Ion Composition Spectrometer on board the CRRES spacecraft, J. Spacecraft and Rockets, 29, 585-591, 1992.

Woch, J., Kremser, G., and Korth, A.: A comprehensive investigation of compressional ULF waves observed in the ring current, J. Geophys. Res.,95, 15 113-15 132, 1990.

Wright, D. M., Yeoman, T. K., and Chapman, P. J.: High-latitude HF Doppler observations of ULF waves: 1 . waves with large 
spatial scale sizes, ann. Geophys., 15, 1548, 1997.

Wright D. M., Yeoman, T. K., Rae, I. J., Storey, J., Stockton-Chalk, A. B., Roeder, J. L., and Trattner, K. J.: Ground-based and Polar spacecraft observations of a giant $(\mathrm{Pg})$ pulsation and its associated source mechanism, J. Geophys. Res., 106, 10 837-10852, 2001.

Yeoman, T. K., Wright, D. M., Chapman, P. J., and Stockton-Chalk, A. B.: High-latitude observations of ULF waves with large azimuthal wavenumbers, J. Geophys. Res., 105, 5453-5462, 2000.
Yeoman, T. K., Mao, T, Lester, M., and Jones, T. B.: A study of Pc5 hydromagnetic waves with equatorward phase propagation, Planet. Space Sci., 40, 797-810, 1992.

Yeoman, T. K. and Wright, D. M.: ULF waves with drift resonance and drift-bounce resonance energy sources as observed in artificially-induced HF radar backscatter, Ann. Geophysicae, 19, 159-170, 2001. 